\title{
Analysis and Comparison of Reordering for Two Factorization Methods (LU and WZ) for Sparse Matrices
}

\author{
Beata Bylina and Jarosław Bylina \\ Department of Computer Science \\ Institute of Mathematics \\ Marie Curie-Sklodowska University \\ Pl. M. Curie-Skłodowskiej 1, 20-031 Lublin, Poland \\ beatas@hektor.umcs.lublin.pl, \\ jmbylina@hektor.umcs.lublin.pl
}

\begin{abstract}
The authors of the article make analysis and comparison of reordering for two factorizations of the sparse matrices - the traditional factorization into the matrices $\mathbf{L}$ and $\mathbf{U}$ as well as the factorization into matrices $\mathbf{W}$ and $\mathbf{Z}$. The article compares these two factorizations regarding: the produced quantity of non-zero elements alias their susceptibility to a fill-in; the algorithms reorganizing matrix (for LU it will be the algorithm AMD but for WZ it will be a modification of the Markowitz algorithm); as well as the time of the algorithms. The paper also describes the results of a numerical experiment carried for different sparse matrices from Davis Collection.
\end{abstract}

\section{Introduction}

It is a very important issue for the numerical linear algebra to solve different linear systems of equations both when the matrix of coefficients is a dense one (that is including few non-zero elements) or when the matrix is sparse. In this paper we deal with a question of solving linear systems with a sparse matrix of coefficients by a factorization of the matrix.

Solving sparse systems demands applying direct or iterative methods. Both kinds of methods have their own merits and flaws. However, in this paper we only handle the direct methods based on Gaussian elimination.

As far as the direct methods are concerned, they demand applying the coefficient matrix factorization into factors of two matrices, e.g. into $\mathbf{L U}, \mathbf{W Z}$ or $\mathbf{Q R}$ as well as into three factors, e.g. into $\mathbf{L D L}^{T}$.

We will assume that $\mathbf{A}$ is a square $(n \times n)$, nonsingular and sparse matrix of not any particular structure. Usually during a factorization of a sparse matrix, matrices which come into existence have far more non-zero elements comparing to the primary matrix. During the matrix A factorization into a product, one has to do with this fill-in problem - consisting in generating additional non-zero elements (except the ones which were non-zero in the matrix A). The fill-in causes a substantial increase in memory requirements and (what comes with that) a worsening 
of a solver performance. Some problems connected to the fill-in are: a reduction of the very fill-in (by some reordering or approximation) and forecasts of positions of non-zeros (for more efficient storing of the matrices' elements).

The fill-in is the reason for applying algorithms and data structures to reduce it and act in due time. A sparse factorization usually consists of two parts. The first part is a reorganization of the matrix and its analysis where a symbolic factorization is done, pointing in anticipation of the places where non-zero elements appear. The second part is a usual numerical sparse matrix factorization into factors.

We can find some examples of this approach - as MUMPS [2] and SuperLU [13. In [3] we can find analysis and comparison of two solvers mentioned above.

In this paper we focus on the first part of the algorithm - that is the reordering.

Reducing of non-zero elements quantity demands applying different permutations of rows and columns (known as reordering). The number of all possible permutations is $n$ ! (for an $n \times n$ matrix) and finding, which of them is the best one, belongs to the class of NP-complete problems.

For structured matrices (like symmetric ones) we can use the Minimum Degree Algorithm [16] or the Nested Dissection [15].

Of course, we do not always know the structure of the matrix so there are heuristic algorithms which reorganize the matrix. Some of them include the Markowitz scheme [14 and the Markowitz scheme with threshold pivoting (for stability) [10. In papers 4 and 5] some other modifications of the Markowitz scheme are considered.

The article considers reordering for the LU and WZ factorizations [9]10,16]18 for a sparse square $(n \times n)$ matrix of not any particular structure.

The article describes and examines a matrix transformation leading to a reduction of non-zero elements in the output matrices $\mathbf{L}$, $\mathbf{U}$ by applying the AMD (Approximate Minimum Degree) algorithm [1] as well as in the output matrices $\mathbf{W}, \mathbf{Z}$ by applying the modified Markowitz algorithm (for the WZ factorization) given by the authors. The aim of the paper is to compare the algorithms in their effectiveness of the fill-in reduction. The performance time of the modified Markowitz algorithm is also considered.

The reasons for choosing AMD is its popularity, accessibility and wide application.

The rest of the paper is organized as follows. Section 2 presents the WZ factorization. Section 3 presents the modifications of the Markowitz scheme for the WZ factorization which ensures the growth of the matrices $\mathbf{W}$ and $\mathbf{Z}$ sparsity and also a factorization stability. Section 4 describes an environment used to numerical experiments conducted for plenty of matrices from Davis Collection and we also present the results of the examination. We will make an analysis, how many non-zero elements we will find in the matrices $\mathbf{L}+\mathbf{U}$ and $\mathbf{W}+\mathbf{Z}$, and also how the AMD algorithm and the modified Markowitz algorithm influence the number of non-zero elements as well as the time of algorithms performance.

In this article we mark the well-known numerical algorithm of the LU factorization simply by LU. The numerical algorithm LU with reordering [1] we mark by $\mathrm{AMD}$ - in the same way as it is marked in the literature. 


\section{WZ Factorization}

The WZ factorization was proposed by Evans and Hatzopoulos [12] as the factorization compatible to SIMD computers. SIMD according to Flynn classification means a Single Instruction stream and a Multiple Data stream, so the SIMD architecture is characterized by multiplexing of processing units. The papers 6 6/11]17 develop and examine the modifications of the WZ factorization method and consider its parallel implementations.

Let $\mathbf{A}$ be a nonsingular matrix. The WZ factorization causes a division of the matrix $\mathbf{A}$ into $\mathbf{W}$ and $\mathbf{Z}$ factors (so that $\mathbf{A}=\mathbf{W Z}$ ) assuming forms which can be described like follows: (for an even $n$ ):

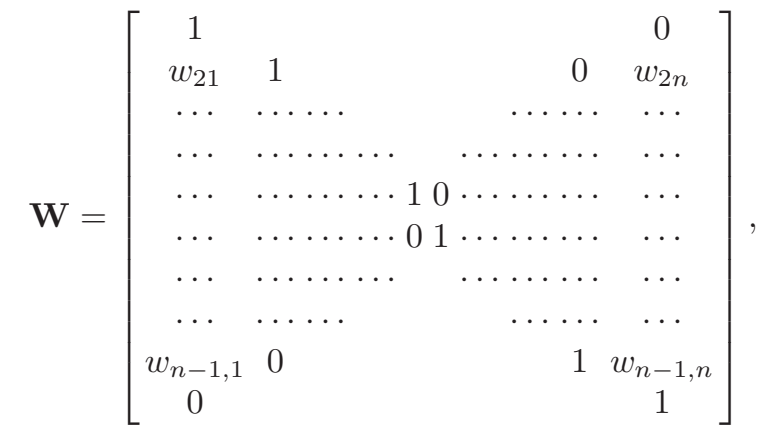

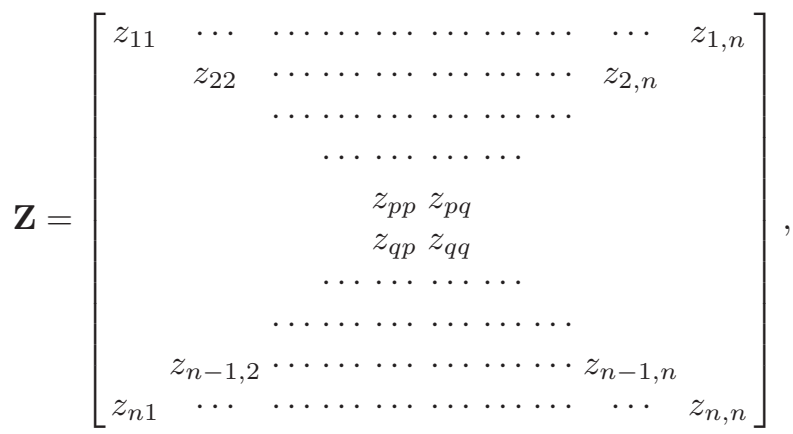

where

$$
m=\lfloor(n-1) / 2\rfloor, \quad p=\lfloor(n+1) / 2\rfloor, \quad q=\lceil(n+1) / 2\rceil .
$$

An example for an odd $n(n=5)$ :

$$
\mathbf{W}=\left[\begin{array}{ccccc}
1 & 0 & 0 & 0 & 0 \\
w_{21} & 1 & 0 & 0 & w_{25} \\
w_{31} & w_{32} & 1 & w_{34} & w_{35} \\
w_{41} & 0 & 0 & 1 & w_{45} \\
0 & 0 & 0 & 0 & 1
\end{array}\right], \quad \mathbf{Z}=\left[\begin{array}{ccccc}
z_{11} & z_{12} & z_{13} & z_{14} & z_{15} \\
0 & z_{22} & z_{23} & z_{24} & 0 \\
0 & 0 & z_{33} & 0 & 0 \\
0 & z_{42} & z_{43} & z_{44} & 0 \\
z_{51} & z_{52} & z_{53} & z_{54} & z_{55}
\end{array}\right]
$$

See also Fig. 1 and Fig. 2 .

The numerical algorithm of the WZ factorization in this article is marked simply by WZ. 

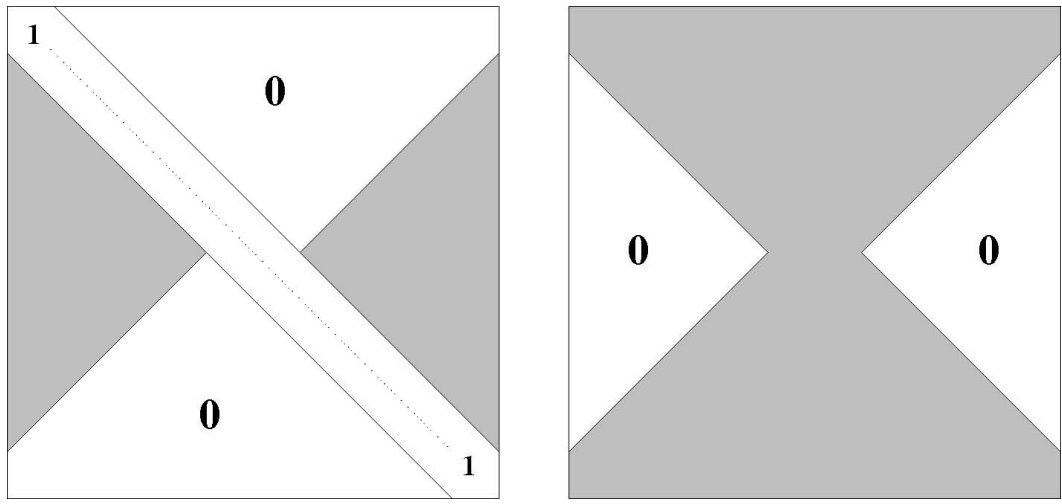

Fig. 1. The form of the output matrices in the WZ factorization (left: $\mathbf{W}$; right: $\mathbf{Z}$ )

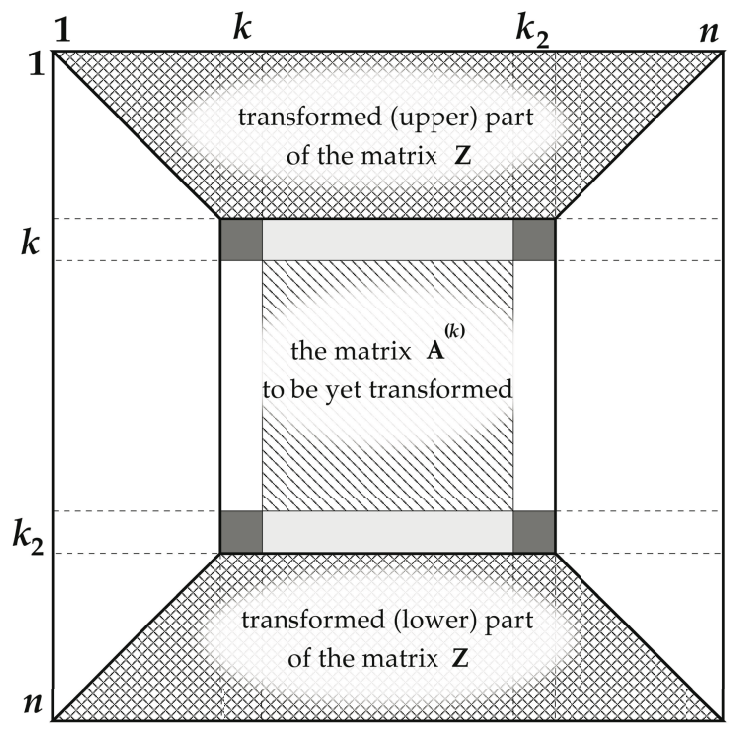

Fig. 2. The $k$ th step of the WZ factorization (actually, of the transformation of the matrix $\mathbf{A}$ into $\mathbf{Z}$ ); here $k_{2}=n-k+1$

\section{Modification of Markowitz Scheme for WZ Factorization}

The original Markowitz scheme was first presented in [14. It consists in a special way of the pivoting - not regarding to the value of pivot element but to the quantity of non-zero elements in rows and columns left to process. The row having the fewest non-zeros is chosen to be swapped with the current row and similarly columns are swapped. Thus, the number of newly generated non-zeros 
(that is the amount of the fill-in) can be reduced significantly. Unfortunately, such an algorithm can lead to a zero pivot and hence make the factorization fail. There are modifications of the Markowitz scheme which ensure success of the factorization (as in 4510).

Here we show a modified Markowitz scheme version for the WZ factorization. Let $\mathbf{A}^{(k)}$ be the matrix obtained from the $k$ th step of the WZ factorization with the size $(n-2 k+2) \times(n-2 k+2)$ (as in Fig. 2), let $r_{i}^{(k)}$ be the number of non-zero values in the $i$ th row of the matrix $\mathbf{A}^{(k)}$. We choose

$$
i_{1}=\arg \min _{i \in\left\{k, \ldots, k_{2}\right\}} r_{i}^{(k)}
$$

and

$$
i_{2}=\arg \min _{i \in\left\{k, \ldots, k_{2}\right\} \backslash\left\{i_{1}\right\}} r_{i}^{(k)} .
$$

Then we swap the $k$ th row with the $i_{1}$ st row and the $k_{2}$ nd row with the $i_{2}$ nd row. (We consider only rows, because in the WZ factorization there would be much more comparisons if we considered also columns because of two pivot rows [instead of only one in LU] and two pivot columns [instead of only one in LU]).

Of course, such swapping can lead to the situation where the determinant

$$
d=a_{k k}^{(k)} a_{k_{2} k_{2}}^{(k)}-a_{k_{2} k}^{(k)} a_{k k_{2}}^{(k)}
$$

(which is the pivot by which we divide in the WZ factorization) will be zero then the continuation of the factorization will not be possible. That is why we must additionally choose $i_{1}$ and $i_{2}$ in the way the determinant $d$ will not equal zero (what is not shown in the above paragraph).

It means that in the modified Markowitz scheme (as in the original one) during each turn of completely external loop there is a need to make many comparisons to choose two rows including the smallest number of non-zero elements.

The algorithm, which consists of the WZ factorization with our modification of the Markowitz algorithm, we mark as MWZ.

\section{Numerical Experiment}

Here we try to compare the performance of some algorithms and study the reordering influence on the number of non-zero elements.

The algorithms' implementation was done using $\mathrm{C}$ language. Data structures to store the matrices $\mathbf{A}, \mathbf{W}, \mathbf{Z}, \mathbf{L}, \mathbf{U}$ were two-dimensional arrays located in RAM. The numerical experiment was done using a Pentium IV $2.80 \mathrm{GHz}$ computer with 1 GB RAM. The algorithms were tested in a GNU/Linux environment and the compilation was done using the compiler $g c c$ with an optimization option -03. Tests were done for matrices from Davis Collection [8].

The tests were done for a set of 40 sparse matrices from different applications. We have not managed to do the WZ factorization for 11 matrices - they were singular. For 14 matrices we needed the WZ factorization with the modified 
Table 1. Test matrices chosen from Davis Collection

\begin{tabular}{rcrrc}
\hline$\#$ & matrix name & matrix size & number of non-zeros & is it symmetric? \\
\hline 1 & Ifat5_e & 14 & 30 & no \\
2 & bcsstko1 & 48 & 224 & yes \\
3 & nos4 & 100 & 347 & yes \\
4 & olm100 & 100 & 396 & no \\
5 & rdb2001 & 2001 & 1120 & no \\
6 & orsirr_1 & 1030 & 6858 & no \\
7 & comsol & 1500 & 97645 & no \\
8 & rdb2048 & 2048 & 12032 & no \\
9 & ex29 & 2870 & 23754 & no \\
10 & rdb3200 & 3200 & 18880 & no \\
11 & rdb5000 & 5000 & 2960 & no \\
12 & uym5940 & 5940 & 85842 & no \\
13 & raefsky5 & 6316 & 167178 & no \\
14 & fp & 7548 & 884222 & no \\
15 & pd & 8081 & 13036 & no \\
\hline
\end{tabular}

Table 2. The comparison of non-zero elements quantity for the algorithms LU, WZ, MWZ, AMD

\begin{tabular}{rrrrr}
\hline$\#$ & LU & WZ & MWZ & AMD \\
\hline 1 & 44 & 44 & 44 & 52 \\
2 & 272 & 272 & 272 & 930 \\
3 & 447 & 447 & 447 & 1164 \\
4 & 639 & 639 & 545 & 494 \\
5 & 7674 & 7368 & 4730 & 3730 \\
6 & 145528 & 207125 & 86392 & 50374 \\
7 & 1176657 & 1101656 & 934350 & 213582 \\
8 & 258298 & 254516 & 114862 & 82234 \\
9 & 217840 & 131951 & 120198 & 127970 \\
10 & 505914 & 274908 & 216135 & 150256 \\
11 & 990394 & 980888 & 409015 & 82234 \\
12 & 2991163 & 2673569 & 1045803 & 656730 \\
13 & 212829 & 226487 & 227613 & 226632 \\
14 & 39967153 & 53861147 & 20092097 & 2875348 \\
15 & 23526 & 23818 & 23088 & 20599 \\
\hline
\end{tabular}

Markowitz scheme (as a kind of pivoting) what enabled the numerical WZ factorization (with no pivoting such factorizations were impossible). Table 1 includes the set of the matrices where the WZ and MWZ algorithms were successfully applied.

Table 2 includes information how many non-zero elements $(n z)$ were created while doing the algorithms WZ, LU, AMD and MWZ. By using data from Davis Collection [8] we placed the number of elements for the matrices created by the algorithm AMD; the results for LU, WZ and MWZ are from the authors' tests. 
Table 3. The comparison of the performance times for the algorithms LU, WZ, MWZ (times given in seconds)

\begin{tabular}{rrrr}
\hline$\#$ & LU & WZ & MWZ \\
\hline 1 & 0.01 & 0.02 & 0.04 \\
2 & 0.01 & 0.03 & 0.07 \\
3 & 0.01 & 0.05 & 0.09 \\
4 & 0.01 & 0.06 & 0.13 \\
5 & 0.01 & 0.10 & 0.20 \\
6 & 2.58 & 1.44 & 1.63 \\
7 & 7.73 & 4.30 & 7.07 \\
8 & 19.14 & 10.41 & 10.59 \\
9 & 51.96 & 28.95 & 29.67 \\
10 & 71.19 & 37.53 & 38.19 \\
11 & 246.53 & 143.66 & 146.96 \\
12 & 411.44 & 237.18 & 248.03 \\
13 & 506.38 & 286.07 & 280.85 \\
14 & 901.20 & 503.96 & 854.40 \\
15 & 1135.50 & 591.44 & 599.64 \\
\hline
\end{tabular}

Table 3 presents time during which the algorithms WZ, LU and MWZ were being done.

The quantities of non-zero elements and the performance times for chosen four matrices are also presented in Fig. 3 and Fig. 4] (They are scaled for every matrix to show the relative changes of the number of non-zeros and the performance time.)

By comparing the algorithms LU and WZ we can notice that the number of non-zero elements generated by these two factorizations is approximately similar. It is possible to find matrices for which the WZ factorization generates fewer nonzero elements than the LU factorization, for example the matrix ex29. But we can find the matrices for which the LU factorization generates fewer non-zero elements, e.g. the matrix fp. For the tested matrices the algorithm WZ generates on the average $2 \%$ fewer non-zero elements than the algorithm LU.

Applying the Markowitz scheme before the further WZ factorization caused a considerable decline of created non-zero elements number. Applying the Markowitz algorithm for the WZ factorization causes an increase of non-zero elements number for the only one matrix among all the tested matrices. For the rest ones, MWZ causes a decrease of non-zero elements number of average $25 \%$ comparing to the WZ algorithm.

Applying the AMD algorithm for the tested matrices considerably reduced the quantity of non-zero elements of average $36 \%$. We managed to find such matrices for which the WZ factorization as well as the MWZ factorization produce fewer non-zero elements than the AMD algorithm, e.g. the matrix ex29. 


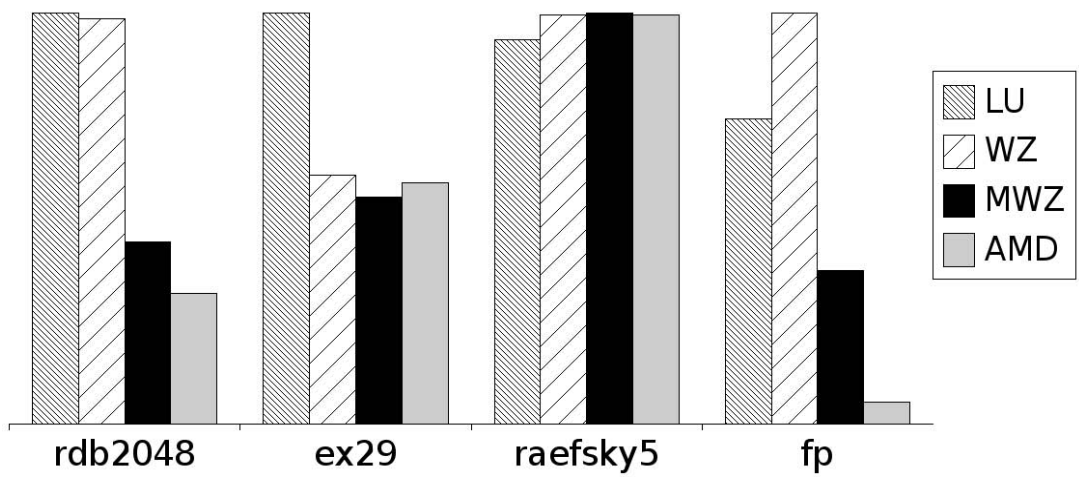

Fig. 3. Relative numbers of non-zeros in the four algorithms for four sample matrices

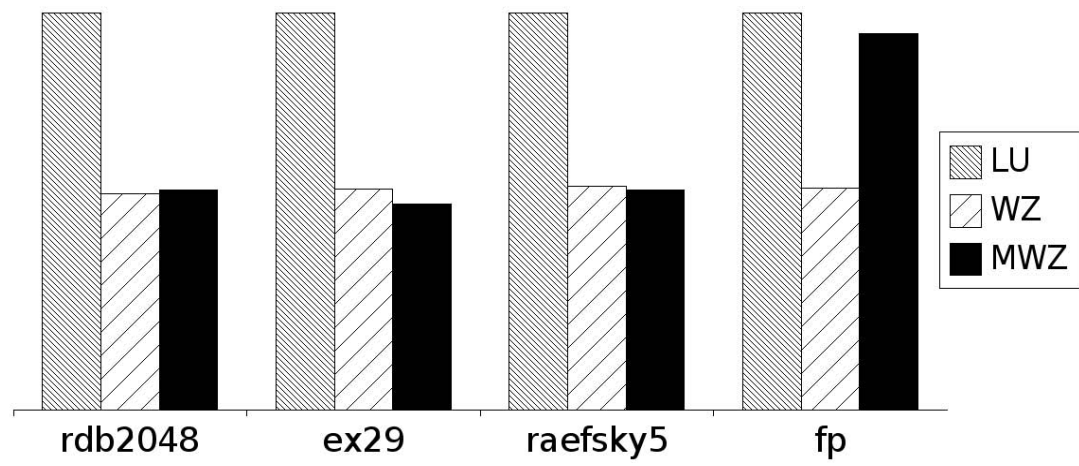

Fig. 4. Relative performance times of the three algorithms for four sample matrices

In the Markowitz scheme comparing to the algorithm which does not use any permutation, time for the tested matrices grows $17 \%$ on the average.. It is worth noticing that the time for $\mathrm{LU}$ is $50 \%$ longer than for WZ.

\section{Conclusions}

In this paper we have presented a detailed analysis and comparison of two reordering schemes. The first, called AMD, is used for the LU factorization; the second - MWZ - proposed by the authors, is used for the WZ factorization. The algorithms' functioning was presented with some sparse matrices taken from concrete engineering applications.

Our analysis is based on experiments with the use of a usual PC. The analysis addresses two aspects of the efficiency of the factorization: the role of the reordering step and the time needed for the factorization. We can summarize 
our observations as follows: there exist matrices for which MWZ (proposed by the authors) is worth using instead of AMD.

Moreover, it appeared that the time of the WZ algorithm was on the average $50 \%$ shorter comparing to the LU algorithm. It results from the fact that loops in the WZ factorization are two times shorter what enables better use of modern processors architecture: threading (possibility to use parallel calculations) and the organization of the processor access to the memory (particularly an optimal use of the multilevel cache memory).

Our future works would research problems of the influence of reordering on the results' numerical accuracy. The other future issue is to name properties of the matrices for which using MWZ is better then using AMD.

Acknowledgments. This work was partially supported within the project Metody $i$ modele dla kontroli zattoczenia $i$ oceny efektywności mechanizmów jakości ustug w Internecie nastepnej generacji (N517 025 31/2997).

This work was also partially supported by Marie Curie-Sklodowska University in Lublin within the project Równoległe algorytmy generacji $i$ rozwiazywania mechanizmów kontroli przeciażenia w protokole TCP modelowanych przy użyciu tańcuchów Markowa.

\section{References}

1. Amestoy, P., Davis, T.A., Duff, I.S.: Algorithm 837: AMD, An approximate minimum degree ordering algorithm. ACM Trans. Math. Soft. 23, 1129-1139 (1997)

2. Amestoy, P.R., Duff, I.S., L'Excellent, J.-I., Koster, J.: A full asynchronous multifrontal solver using distributed dynamic scheduling. SIAM J. Matr. Anal. Apl. 23(1), 15-41 (2001)

3. Amestoy, P.R., Duff, I.S., L'Excellent, J.-I., Li, X.S.: Analysis and Comparison of Two General Sparse Solvers for Distributed Memory Computers. ACM Trans. Math. Soft. 27(4), 388-421 (2001)

4. Amestoy, P., Li, X.S., Ng, E.G.: Diagonal Markowitz Scheme with Local Symmetrization. Report LBNL-53854 (2003); SIAM. J. Matr. Anal. Appl. 29, 228 (2007)

5. Amestoy, P., Pralet, S.: Unsymmetric Ordering Using a Constrained Markowitz Scheme. SIAM J. Matr. Anal. Appl.; Report LBNL-56861 (submitted, 2005)

6. Bylina, B., Bylina, J.: The Vectorized and Parallelized Solving of Markovian Models for Optical Networks. In: Bubak, M., van Albada, G.D., Sloot, P.M.A., Dongarra, J. (eds.) ICCS 2004. LNCS, vol. 3037, pp. 578-581. Springer, Heidelberg (2004)

7. Chandra Sekhara Rao, S.: Existence and uniqueness of WZ factorization. Parall. Comp. 23, 1129-1139 (1997)

8. Davis, T.: University of Florida Sparse Matrix Collection. NA Digest 92(42) (1994), NA Digest 96(28) (1996), and NA Digest 97(23) (1997), http://www.cise.ufl.edu/research/sparse/matrices

9. Duff, I.S.: Combining direct and iterative methods for the solution of large systems in different application areas. Technical Report RAL-TR-2004-033 (2004)

10. Duff, I.S., Erisman, A.M., Reid, J.: Direct Methods for Sparse Matrices. Oxford University Press, New York (1986) 
11. Evans, D.J., Barulli, M.: BSP linear solver for dense matrices. Parall. Comp. 24, 777-795 (1998)

12. Evans, D.J., Hatzopoulos, M.: The parallel solution of linear system. Int. J. Comp. Math. 7, 227-238 (1979)

13. Li, X.S., Demmel, J.W.: A scalable sparse direct solver using static pivoting. In: Proceedings of the Ninth SIAM Conference on Parallel Processing for Scientific Computing (1999)

14. Markowitz, H.M.: The elimination form of the inverse and its application to linear programming. Management Science 3, 255-269 (1957)

15. Reid, J., Duff, I.S., Erisman, A.M.: On George's nested dissection method. SIAM J. Numer. Anal. 13, 686 (1976)

16. Tinney, W.F., Walker, J.W.: Direct solution of sparse network equations by optimally ordered triangular factorization. Proc. IEEE 55, 1801-1809 (1967)

17. Yalamov, P., Evans, D.J.: The WZ matrix factorization method. Parall. Comp. 21, 1111-1120 (1995)

18. Zlatev, Z.: On some pivotal strategies in Gaussian elimination by sparse technique. SIAM J. Numer. Anal. 17, 18-30 (1980) 